\title{
The interplay of framework instability and electron-phonon coupling in a CDW system, the monophosphate tungsten bronze family.
}

\author{
A. Minelli ${ }^{1}$, E. Duverger-Nedellec ${ }^{2}$, A. Pautrat ${ }^{3}$, O. Pérez ${ }^{3}$, M. Mihalkovic ${ }^{4}$, M. De Boissieu ${ }^{5,6}$, A. Bosak ${ }^{7}$, \\ A. Goodwin ${ }^{1}$ \\ ${ }^{1}$ University of Oxford, Oxford, United Kingdom, \\ ${ }^{2} I C M C B, C N R S$, Université de Bordeaux, UMR 5026, F-33600 Pessac, France, \\ ${ }^{3}$ Laboratory CRISMAT, UMR 6508 CNRS, ENSICAEN 6 Boulevard du Marechal Juin, F-14050 Caen Cedex 4, France, \\ ${ }^{4}$ Institute of Physics, Slovak Academy of Sciences, Dúbravskà cesta 9, Bratislava 84511, Slovak Republic, \\ ${ }^{5}$ Université Grenoble Alpes, SIMaP, F-38000 Grenoble, France, \\ ${ }^{6} \mathrm{CNRS}$, SIMaP, F-38000 Grenoble, France, \\ ${ }^{7}$ European Synchrotron Radiation Facility, 6 rue Jules Horowitx, F-38000 Grenoble, France
}

\section{arianna.minelli@.chem.ox.ac.uk}

The tungsten bronzes are low-dimensional transition metal oxides of great interest for their electronic instabilities. They show exotic physical properties such as superconductivity and charge density wave (CDW) phases. An important subfamily is $\left(\mathrm{PO}_{2}\right)_{4}\left(\mathrm{WO}_{3}\right)_{2 \mathrm{~m}}$, which is interesting for its optical/magnetic behaviours, where the band filling and CDW phases coupled in different way with the lattice. These properties can be tuned by $m$, the thickness of the perovskite-like $\mathrm{WO}_{6}$-octahedra block [1].

To understand the electronic instabilities, correlated to the nesting properties of the Fermi surface and the consequent CDW phases, we used the combination of two techniques: diffuse scattering (DS) and inelastic x-ray scattering (IXS). This allows rapid identification of the nature of diffuse features in the patterns and the study of the lattice dynamics. Three different members are chosen in order to show the evolution of the behaviour in the family. In this context, we will focus on the lattice dynamics and framework instability. The first member, $m=2$, presents a quasi-1D instability given by the $\mathrm{WO}_{3}$-octahedra zig-zag chains, which are isolated by the phosphates. A CDW phase is found, $\mathrm{T}_{\mathrm{C}}=270 \mathrm{~K}$, and it is linked to a rigid-body motion. Different behaviour can be found in the members $m=6$ and 8 , where the instability is found in the $\mathrm{WO}_{3}$ slabs, realised as correlated displacements of tungsten atoms along the octahedral 4-fold axis direction. The three members show different diffuse patterns, figure 1. The results are linked to the lattice dynamics behaviour, which present a Kohn anomaly above the transition temperature, however as predicted from the diffuse results, has a different $\mathbf{Q}$ - and temperature-dependence in each member.

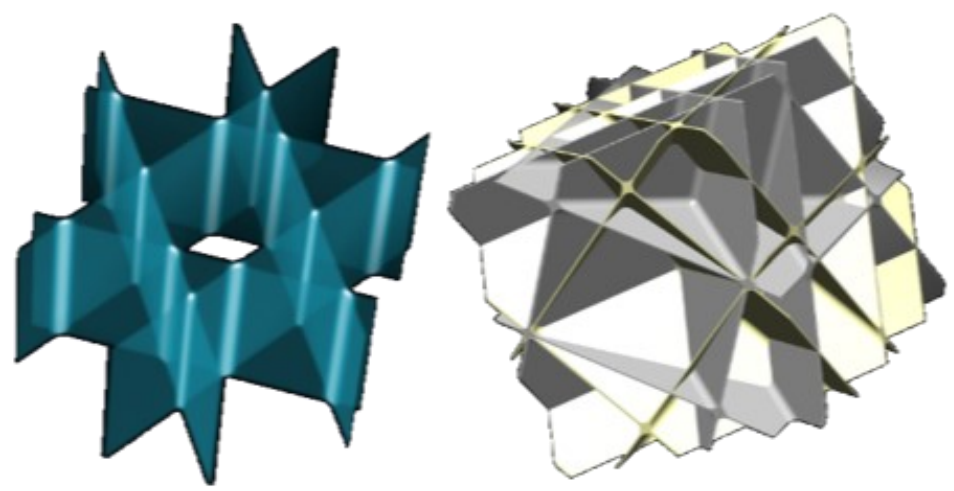

a)

b)

c)

Figure 1. The pre-transitional diffuse patterns for three members, a) $\mathrm{m}=2$; $\mathrm{b}$ ) $\mathrm{m}=6$ and 3) $\mathrm{m}=8$, are represented in this reconstruction.

[1] P. Roussel et al., Acta Cryst. B 57 (2001) 603-632

Keywords: monophosphate tungsten bronze; lattice dynamics; framework instabilities 